Memoirs of The College of Science, University of Kyoto, Series A

Vol. XXX, Mathematics No. 1, 1956.

\title{
On Riemann's period relations on open Riemann surfaces
}

\author{
By \\ Yukio KuSUNOKI \\ (Received April 12, 1956)
}

Introduction.

The theory of Abelian differentials of the first kind on abstract open Riemann surfaces was first developped in 1940 by R. Nevanlinna [13]. This theory was established for parabolic Riemann surfaces by considering the complete orthogonal system of such differentials, and was completed by Virtanen [23] in 1950 for general Riemann surfaces. On the other hand, in view of period relations Ahlfors [1], Virtanen [22] treated this theory for parabolic Riemann surfaces, where Riemann's bilinear relation plays a fundamental role. For the case of hyperelliptic surfaces of infinite genus this relation was investigated in detail by Hornich [6], P. J. Myrberg [12] and recently Pfluger [17].

In the present paper also the problem on the periods of Abelian integrals on an abstract open Riemann surface will be treated." Ahlfors [1] proved the existence of an exhaustion and corresponding canonical homology basis of $R \in O_{i}$ (class of parabolic Riemann surfaces) such that for any two harmonic differentials $d u_{1}, d u_{2}$ with finite Dirichlet integrals the mixed Dirichlet integral $D_{R}\left(u_{1}\right.$, $u_{2}$ ) is equal to

$$
D_{k}\left(u_{1}, u_{2}\right)=\lim _{n \rightarrow \infty} \sum_{i=1}^{k_{n}}\left(\int_{A_{i}} d \bar{u}_{1} \int_{B_{i}} d \bar{u}_{2} *-\int_{A_{i}} d \bar{u}_{2} * \int_{B_{i}} d \bar{u}_{1}\right),
$$

where $d \bar{u}_{1}$ and $d \bar{u}_{2}^{*}$ are the modified quantities of $d u_{1}$ resp. $d u_{2}{ }^{*}$ (conjugate harmonic differential of $d u_{2}$ ) which depend on the exhaustion.

To obtain the corresponding formula which is expressed by

1) The principal results in this paper have been announced and partly proved in my notes [8], [9]. 
the periods of $d u_{1}$ and $d u_{2}{ }^{*}$ I have to impose further some conditions, that is, for certain restricted class $O^{\prime}$ of $O_{G}$, Riemann's first and second (bilinear) relations with an infinite number of their periods will be obtained $(\S 2,3)$.

In connexion with $O^{\prime}$ we shall consider another subclass $O^{\prime \prime}$ of $O^{\prime}$. These classes are defined by the extremal length. In $\S 1$ we shall study their properties, above all, those concern with the problem of limit (at ideal boundary) of bounded harmonic functions.

Finally I shall extend Riemann's second relation to the ultimate form when $d u_{1}$ (or $d u_{2}{ }^{*}$ ) has only a finite number of non vanishing periods, that is, it will be established for Riemann surfaces $R$ of class $O_{H D}$ (on which no harmonic function with finite Dirichlet integral exists). On the other hand, it will be also obtained when $d u_{1}$ and $d u_{2}{ }^{*}$ have only a finite number of non vanishing $A$ periods, if we impose some conditions on the structure of $R \in O_{m \nu}$. $(\S 3)$.

\section{$\S 1$. Two classes of Riemann surfaces.}

1. Extremal length.-To define the subclasses of class $O_{G}$ we shall start with preliminaries on the extremal length on Riemann surfaces (cf. Ahlfors, Beurling [3], Hersch [5], Ohtsuka [16]). Now let $R$ be an arbitrary Riemann surface and $G$ be a domain on $R$. We consider a system of curves $\{c\} \neq \phi(\phi$ : empty set) on $G$ each curve of which consists of a finite or a countable number of curves on $G$. Let $(P)$ be the set of non-negative covariants $\mu$ defined on $G$, i.e. $\mu(z)|d z|$ is non-negative invariant metric under the transformations of local parameter $z$ at $p$, such that

$$
L\left(\rho, ; c_{i}^{\prime}\right)=\inf _{c \in c^{\prime} ;} \int_{r} \rho(z)|d z|
$$

(1. 1)

$$
A(\rho)=\iint_{\sigma_{i}} l^{2} d x d y, \quad z=x+i y
$$

are not simultaneously 0 or $\infty$.) Then the extremal length with

\footnotetext{
2) $\int$ is the lower integral, $\bar{\iint}$ is the upper integral in Darboux's sense.
} 
respect to $; c\}$ is defined by

$$
\lambda_{\left(r^{\prime}\right)}\{c\}=\sup _{\rho \in\left(I^{\prime}\right)} \frac{L\left(\rho,\left\{c_{i}^{\prime}\right)^{2}\right.}{A(\rho)} \geqq 0 .
$$

We take $\lambda_{(P)} ; c:=0$ if $(P)$ is empty.

Next we consider another class $(Q)$ of non-negative covariants $\rho$ such that, for any curve $c \epsilon\} c\}$,

$$
\int_{c} \rho(z)|d z| \geqq 1 \text {. }
$$

Then another simple definition of the extremal length is

$$
\frac{1}{\left.\lambda_{(Q)} \backslash c\right\}}=\inf _{\mu \in(Q)} A(\rho) \text {. }
$$

We say that $\rho$ is admissible for $i c ;$ when (1.3) is satisfied. If there is no admissible covariant, i.e. $\left.(Q)=\phi, \lambda_{(q)} ; c\right\}=0$.

Proposition 1.-

$$
\left.\dot{\lambda}_{(P)} ; c\right\}=\lambda_{(Q)}\{c\} .
$$

In the following therefore we denote this common value by $\lambda ; c\}$.

Proof. First we suppose $(P) \neq \phi$.

(I) The case where $L(\rho,\{c\})<\infty$ for all $\rho \epsilon(P)$.

(i) When there exists at least one $\mu \epsilon(P)$ such that $0<L(\rho,\{c\})$ $<\infty$, we choose a constant $k$ such that $L\left(\rho^{\prime},\{c\}\right)=1, \imath^{\prime}=k_{l^{\prime}} \in(Q)$. Then it follows easily

$$
\left.0 \leqq \lambda_{(P)}\{c\}=\sup _{P^{\prime}} 1 / A\left(o^{\prime}\right) \leqq \lambda_{(q)} ; c\right\}
$$

Now in general $(Q)=P_{1} \cup P_{2} \cup P_{3}$ where $P_{1}=\left\{p_{1} ; 1 \leqq L\left(\mu_{1}, ; c\right\}\right)<\infty\{$, $P_{2}=\left\{\mu_{2} ; L\left(\rho_{2},\left\{c_{\}}\right)=\infty, A\left(\mu_{2}\right)<\infty\right\}\right.$ and $P_{3}=\left\{p_{3} ; L\left(\rho_{3}, ; c\right\}\right)=A\left(p_{3}\right)$ $=\infty\} . \quad P_{1} \subset(P), P_{2} \subset(P)$, but since $P_{2}=\phi$ in the present case, we have conversely

(1. 7) $\left.\quad \lambda_{(P)} ; c\right\} \geqq \sup _{\rho \in P_{1}} \frac{L(\rho ;\{c\})^{2}}{A(\rho)} \geqq \sup _{\rho \in \mu_{1}} \frac{1}{A\left(\rho^{\prime}\right)}=\sup _{\rho \in P_{1} \cup P_{3}} \frac{1}{A(\mu)}=\lambda_{(\rho)}\{c\}$.

(ii) If $L\left({ }^{\prime \prime},\{c\}\right)=0$ for all $" \prime \epsilon(P)$, then $(Q)=\phi$ or for any $\rho \in(Q) L(\rho)=A(\rho)=\infty$, hence $\lambda_{(P)}\{\boldsymbol{c}\}=\lambda_{(2)} ; \boldsymbol{c} ;=0$ by definition.

(II) The case where there exists at least one $\rho_{0} \epsilon(P)$ for which $L\left(o_{0},\{c\}\right)=\infty$.

Then $A\left(\gamma^{\prime}{ }_{0}\right)<\infty$ and $\left.\lambda_{(r)} ; c\right\}=\infty$. Since the covariants $\rho_{n}=\mu_{0} / n$ $(n=1,2, \ldots)$ are admissible for $(Q)$, 


$$
0 \leqq \dot{\lambda}_{(())} ; c^{\prime-1}=\inf _{\rho \in((l)} A(o) \leqq \lim _{n \rightarrow \infty} A\left(\rho_{0}\right) / n^{2}=0
$$

Therefore

$$
\left.\lambda_{(r)}\{c\}=\lambda_{(r)} ; c\right\}=\infty \text {. }
$$

Now if $(P)=\phi$, then $i_{(P)}\left\{c_{i}=0\right.$ and for any non-negative covariant $\rho L\left(f^{\prime},\{c\}\right)$ and $A\left({ }^{\prime \prime}\right)$ are simultaneously zero or $\infty$. Hence it is proved that $\left.\lambda_{(m)} ; c_{i}=\lambda_{(())} ; c\right\}=0$, q.e.d.

We shall use the following properties of the extremal length.

Proposition 2

(i) If $\left\{c_{1}\left\{\subset\left\{c_{2}\right\}\right.\right.$, then $\left.\left.\left.\lambda c_{1}\right\} \geqq \lambda\right\} c_{2}\right\}$.

$$
\begin{gathered}
\text { (ii) } \left.\lambda\left\{\left\{c_{1}\right\} \cup\left\{c_{2}\right\}\right\}^{-1} \leqq \lambda\left\{c_{1}\right\}^{-1}+\lambda, c_{2}\right\}^{-1} \text {. } \\
\text { (iii) If }\left\{c_{1}\right\} \subset G_{1} \subset G,\left\{c_{2}\right\} \subset G_{2} \subset G \text { and } G_{1} \cap G_{2}=\phi \text {, then } \\
\left.\lambda\left\{\left\{c_{1}\right\} \cup\left\{c_{2}\right\}\right\}^{-1}=\lambda, c_{1}\right\}^{-1}+\lambda\left\{c_{2}\right\}^{-1} \text {. }
\end{gathered}
$$

(iv) If every curve c $\epsilon\{c\}$ contains at least one $c_{1} \epsilon\left\{c_{1}\right\}$ and one $c_{2} \epsilon\left\{c_{2}\right\}$ where $\left\{c_{1}\right\} \subset G_{1} \subset G,\left\{c_{2}\right\} \subset G_{2} \subset G$ and $G_{1} \cap G_{2}=\phi$, then

$$
\left.\lambda_{i} c_{1}\right\}+i c_{2} i \leqq \lambda_{i} c_{\}} \text {. }
$$

2. In the following we take $G=R$ without loss of generality. Let $B$ be a union of a finite number of disjoint ring domains $B_{i}$ on $R$ each of which has boundaries $\alpha_{i}$ and $\beta_{i}$ which consist of a finite number of disjoint analytic Jordan closed curves respectively. Let $\{c\}$ be the set of closed curves $c$ on $B$ such that $c=\sum_{i} c_{i}, c_{i}$ is homologous to $\alpha_{i}$, i.e. $c_{i} \sim \alpha_{i} \sim \beta_{i}$. Now let $\left\{c^{*}\right\}$ be a subset of $\{c\}$ which consist of analytic Jordan closed curves. Suppose $\{\tilde{c}\}$ and $\left\{\tilde{c}^{*}\right\}$ denote the corresponding sets for the union of curves in $B_{i}$ which connect $\alpha_{i}$ to $\beta_{i}$. By Prop. 2 (i) $\left.\left.\lambda\left\{c_{\}} \leqq \lambda ; c^{*}\right\}, \lambda ; \tilde{c}\right\} \leqq \lambda ; \tilde{c}^{*}\right\}$, but we have

Proposition 3.

$$
\left.\lambda\{c\}=\lambda i c^{*}\right\}=D_{n}(\omega)=\frac{1}{\lambda ; \tilde{c}\}}=\frac{1}{\left.i ; \tilde{c}^{*}\right\}} .
$$

where $D_{B}(\omega)$ stands for the Dirichlet integral over $B$ of the harmonic measure $\omega$ (in $B$ ) with resp. to $\beta$, i.e. $\omega=\omega_{i}$ in $B_{i}$, $\omega_{i}$ is harmonic measure of $B_{i}$ with resp. to $\beta_{i}$.

Proof. Let $L_{\lambda}$ be the level curve $(1=\lambda, 0 \leqq \lambda \leqq 1$ except a finite number of $\lambda$ for which $L_{\lambda}$ contain the points where grad $\omega=0$. Obviously $L_{\lambda} \in\left\{c *\{\subset ; c\}\right.$. Suppose $\rho$ is admissible for $\left\{c^{*}\right\}$. Since $Q=\omega+i \omega^{*}$ is considered as a uniformizer at $B$ with a finite number of suitable slits $I^{\prime}: \omega^{*}=$ const., 
On Riemann's period relations on open Riemann surfaces

$$
1 \leqq \int_{L_{\lambda}} \rho d \omega^{*}, 0 \leqq \lambda \leqq 1
$$

Since $\int_{L_{\lambda}} d \omega^{*}=\int_{L_{1}} d \omega^{*}=D_{B}(\omega)$, by using Schwarz's inequality and integrating we have

Therefore

$$
1 \leqq D_{B}(\omega) \overline{\iint_{B} r^{2} d \omega d \omega^{*}}
$$

(1. 9) $\quad D_{B}(\omega)^{-1} \leqq \inf _{p} \bar{\int} \int_{B} r^{2} d \omega d \omega^{*} \leqq \inf _{p} A(\rho)=\lambda\left\{c^{*}\right\}^{-1} \leqq \lambda\left\{c^{\prime-1}\right.$.

On the other hand,

(1. 10) $\tilde{\rho}(p(\zeta))=\left\{\begin{array}{l}D_{n}^{-1}(\omega)|d \Omega / d \zeta|, p(\zeta) \in B^{\prime}, B^{\prime}=B-\Gamma \\ 0, \quad p(\zeta) \epsilon R-B^{\prime}\end{array}\right.$

is admissible for $\{c\} \supset\left\{c^{*}\right\}$. Hence we have conversely

$$
\lambda\left\{c^{*}\right\}^{-1} \leqq \lambda\left\{c^{-1} \leqq A(\tilde{\rho})=D_{B}(\omega)^{-1} .\right.
$$

We can prove analogously that $D_{B}(\omega)=\lambda\left\{\tilde{c}_{\}}^{-1}=\lambda\left\{\tilde{c^{*}}\right\}-1\right.$.

3 . In the following we say that $K$ is a cumpact domain with analytic boundaries when $K$ is a compact domain and its boundary $\partial K$ consists of a finite number of disjoint analytic Jordan closed curves. Now we consider the system of curves $\{C\} \subset R-R_{0}\left(R_{0}\right.$ is the image of a parameter disc), such that $\boldsymbol{C} \epsilon\left\{\boldsymbol{C}_{\}}\right.$consists of a finite number of disjoint analytic Jordan closed curves which is homologous to $\partial R_{0}$, i.e. $C \sim \partial R_{0}$. Let $\{\Gamma\}$ be a system of analytic curves in $R-R_{0}$ each of which extends from $\partial R_{0}$ to the ideal boundary $\mathfrak{\Im}$ of $R$. This means as follows. The ideal boundary $\mathfrak{J}$ of $R$ is the set of ideal boundary elements $\alpha$ which is defined as follows (Stoilow [21]) : Let $\left\{Q_{n}\right\}$ be a sequence of domains on $R$ such that

(1) The relative boundary $C_{n}$ of $\Omega_{n}$ consists of an analytic Jordan closed curve on $R$.

(2) $\Omega_{1} \supset \Omega_{2} \supset \cdots \supset \Omega_{n} \supset \cdots, \bar{\Omega}_{1} \neq R^{3)}$

(3) $\bigcap_{n=1}^{\infty} \Omega_{n}=\phi$.

Then we say $\left\{\Omega_{n}\right\}$ defines an ideal boundary element $\alpha$. We find that $\Omega_{n}$ are non-compact by (3) and $C_{n}$ divides $R$ into two disjoint

3) The barred letter stands for the closure of set. 
parts $\Omega_{n}$ and $R-\bar{\Omega}_{n}$ (we shall frequently write such a curve or cycle as $\sim 0(\bmod \Im)$ i.e. when it is the boundary of an infinite 2-dimensional chain). Two such sequences $\left\{\Omega_{n}\right\}$ and $\left\{\Omega_{n}{ }^{\prime}\right\}$ are called equivalent each other if for given $i, j$ there exist $k, l$ such that

$$
\Omega_{i}^{\prime} \supset \Omega_{k}, \quad \Omega_{j} \supset \Omega_{l}^{\prime} \text {. }
$$

We understand that two equivalent sequences determine the same boundary element. Next we say that a sequence of points $\left\{P_{n}\right.$ i or curves $\left\{\gamma_{n}\right\}$ on $R$ tend to $\alpha$ (or $\Im$ ) resp. to $\Im$ according as all $P_{n}$ or $\gamma_{n}$ except a finite number of points or curves belong to every $\Omega_{m}$ (or $R-\bar{R}_{m}$ ) resp. to $R-\bar{R}_{m}$ and that a curve $\Gamma$ extends to $\mathfrak{I}$

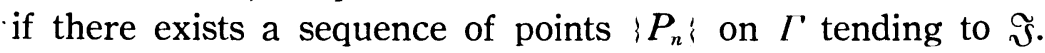

PROPOSITION 4 (Ohtsuka [16]) $-R$ is of parabolic type (Nullrand) if and only if

$$
\lambda\left\{C_{\}}=\lim _{n \rightarrow \infty} \lambda\left\{C^{n} ;=1 / \lambda\{\Gamma\}=\lim _{n \rightarrow \infty} 1 / \lambda\left\{\Gamma^{n}\right\}\right.\right.
$$

is equal to zero, where $\left\{C^{n} ;\right.$ is the subset of $\{C$; lying inside of $G_{n}=R_{n}-R_{0}$, here $\left\{R_{n}\right\}$ is the usual exhaustion of $R$, and $\left\{\Gamma^{n}\right\}$ is the set of analytic curves in $G_{n}$ connecting $\partial R_{0}$ to $\partial R_{n}$.

Proof. Since $\left\{C^{n}\right\} \subset\left\{C^{n+1}\right\} \subset\{C\}$, by Prop. 2 (i), 3 we have

$$
\left.\lim _{n \rightarrow \infty} \lambda C^{n}\right\}=\lim _{n \rightarrow \infty} d_{n} \equiv d \geqq \lambda\{C\} \geqq 0
$$

where $d_{n}=D_{G_{n}}\left(\omega_{n}\right)$ and $\omega_{n}$ is the harmonic measure of $G_{n}$. Hence if $d=0, d=\lambda\{C\}=0$. If $d>0$, since $\omega_{n}$ converge uniformly to a non-constant harmonic function $\omega$ on every compact set in $R-\bar{R}_{0}$, hence we have for any $C \in\{C\}$

$$
\int_{c} d \omega^{*}=\lim _{n \rightarrow \infty} \int_{C} d \omega_{n}^{*}=\lim _{n \rightarrow \infty} d_{n}=d .
$$

Therefore the covariant

$$
\bar{\rho}(p(\zeta))= \begin{cases}d^{-1}|d \Omega / d \zeta|, \quad p(\zeta) \epsilon R-R_{0}-\Gamma, \quad & \Omega=\omega+i \omega^{*} \\ 0, & p(\zeta) \in R_{0}+\Gamma\end{cases}
$$

where $\Gamma^{\prime}$ denotes a countable number of suitable slits $\omega^{*}=$ const. through the points where $Q^{\prime}=0$, is admissible with respect to $\{C$ \} and

$$
\lambda\{C\}^{-1} \leqq \iint_{R}^{-2} \rho^{-2} d x d y=d^{-2} \iint_{R-R_{0}} d \omega d \omega^{*} \leqq d^{-1}
$$


On Riemann's period relations on open Riemann surfaces

therefore together with (1.12) we obtain

$$
d=\lambda\{C\}=\lim _{n \rightarrow \infty} \lambda\left\{C^{n}\right\} .
$$

We can also prove easily that $\lambda\{\Gamma\}=d^{-1}$. On the other hand, according to R. Nevanlinna's theorem [13], $R$ is of parabolic type if and only if $d=0$, q.e.d.

4. Now we shall consider a subset $\{r\}$ of $\{C\}$ which contains an infinite number of curves $\epsilon\{C\}$ tending to $\mathfrak{\Im}$. Let $\left\{\gamma^{*}\right\}$ be the complementary set of $\{r\}$ with respect to $\{C\}$. Since

$$
\begin{aligned}
& \lambda\{C\}^{-1} \leqq \lambda\{r\}^{-1}+\lambda\left\{\gamma^{*}\right\}^{-1}, \\
& 0 \leqq \lambda ; C\} \leqq \lambda\{r\}, \quad 0 \leqq \lambda\{C\} \leqq \lambda\left\{\gamma^{*}\right\},
\end{aligned}
$$

we have

PROPOSITION 5.- $R$ is of parabolic type if and only if at least $\lambda ; \gamma^{\prime}:=0$ or $\lambda\left\{\gamma^{*}\right\}=0$ holds.

PROPOSITION 6.-Let $K \supset R_{0}$ be a compact domain with analytic boundaries. Let $\left\{\gamma_{K}\right\}=\left\{\gamma_{K} ; \gamma_{K} \in\{\gamma\}, \gamma_{K} \cap K=\phi\right\}^{4)}$ and $\left\{\gamma_{K}^{*}\right\}$ be the complementary set of $\left\{\gamma_{K^{\prime}}\right.$ with respect to $\{r\}$. In order that $\left.\lambda i \gamma_{\}}\right\}=0$ it is necessary and sufficient that $\left.\lambda i \gamma_{K}\right\}=0$.

Proof. Since $0 \leqq \lambda\{r\} \leqq \lambda\left\{\gamma_{K}\right\}$, it is sufficient. Therefore it is enough to prove $\lambda\left\{\gamma_{K}^{*}\right\}>0$, since $\lambda\{\gamma\}^{-1} \leqq \lambda\left\{\gamma_{K}\right\}^{-1}+\lambda\left\{\gamma \gamma_{K}^{*}\right\}^{-1}$. Let $K_{0}$ be a compact domain with analytic boundaries containing $K$ completely and $K_{0}-R_{0}=K_{0}{ }^{*}$. Let $\omega(p)$ be the harmonic measure of $K_{0}^{*}$. Put

$$
0<\max _{p \in \partial K}(1)(p)=m<1, \int_{\partial r_{0}} d \omega^{*}=d>0 .
$$

The covariant

$$
\tilde{\rho}(p(\zeta))=\left\{\begin{array}{l}
d^{\prime}|d \varrho / d \zeta|, d^{\prime}=1 / \min (d, 2(1-m)), p(\zeta) \in K_{0}{ }^{*}-\Gamma^{\prime} \\
0, \quad p(\zeta) \in R-K_{0}{ }^{*}+\Gamma
\end{array}\right.
$$

where $Q=\omega+i \omega^{*}$ and $I$ denotes slits (cf. (1.10)), is admissible for $\left\{r_{K}^{*}\right\}$ because

$$
\int_{-r_{K}^{*}} \tilde{\rho}|d z|=d^{\prime} \int_{r_{K}^{*} \cap K_{0}^{*}}|d \Omega|\left\{\begin{array}{l}
=d^{\prime} \int_{r_{K}^{*}}|d \Omega| \geqq d^{\prime}\left|\int_{r_{K}^{*}} d \omega^{*}\right|=d^{\prime} d \geqq 1, \text { if } \gamma_{K}^{*} \subset K_{0}^{*} . \\
\geqq d^{\prime} \int_{r_{K}^{*} \cap K_{0}^{*}} \mid d\left(\omega \mid \geqq d^{\prime} 2(1-m) \geqq 1, \text { if } r_{K}^{*} \quad K_{0}^{*} .\right.
\end{array}\right.
$$

4) In the following we shall use also such a notation. 
Therefore we have

$$
\lambda\left\{\gamma_{K}^{*}\right\}^{-1} \leqq \iint_{R} \tilde{\rho}^{2} d x d y=d^{\prime 2} d<\infty, \quad \text { q.e.d. }
$$

PROPOSITION 7.- Suppose that $\varphi_{1}$ and $\varphi_{0}$ are any two nonnegative covariants sequare integrable over $R-K$ ( $K$ is a compact domain with analytic boundaries). If $\lambda\left\{\gamma_{j}=0\right.$, then there exists a sequence of curves $\gamma_{n} \in\{r\}\left(\gamma_{n} \cap K=\phi\right)$ tending to the ideal boundary $\mathfrak{I}$ of $R$ such that

$$
\int_{\gamma_{n}} \varphi_{1}|d z| \int_{\gamma_{n}} \varphi_{s}|d z| \rightarrow 0 \text { for } n \rightarrow \infty .
$$

Proof. Now we assume that for any $\gamma_{K} \epsilon\left\{\gamma_{K}\right\}$

$$
\int_{-r_{K^{*}}} \varphi_{1}|d \dot{z}| \int_{\gamma_{h^{*}}} \varphi_{2}|d z| \geqq \eta>0 \text {. }
$$

Since $\varphi_{1}$ and $\varphi_{2} \geqq 0$, we have $\int_{-} \varphi_{h^{\prime}}|d z| \geqq \sqrt{\eta} \overline{\text { or }} \int_{-} \varphi_{h^{\circ}}|d z| \geqq \sqrt{\eta}$. Let $\left\{\gamma_{K}^{i}\right\}=\left\{\gamma_{K}^{i} ; \int_{-} \varphi_{r^{i}}|d z| \geqq \sqrt{n}, r_{K}^{i} \in\left\{\gamma_{K}\right\}\left\{(i=1,2)\right.\right.$, then $\left\{\gamma_{K}\right\}=\left\{\gamma_{K}{ }^{1}\right\} \cup\left\{\gamma_{K}{ }^{2}\right\}$, hence $\lambda\left\{\gamma_{K}\right\}^{-1} \leqq \lambda\left\{r_{K}\right\}^{-1}+\lambda\left\{\gamma_{K^{2}}\right\}^{-1}$. Since $\lambda\left\{\gamma_{K}\right\}=0$ (Prop. 6), it follows that $\lambda\left\{r_{K}{ }^{1}\right\}=0$ or $\lambda\left\{r_{\alpha^{2}}{ }^{2}\right\}=0$, e.g. $\lambda\left\{r_{K}{ }^{1}\right\}=0$. Then the covariant $\psi=$ $\varphi_{1} / \sqrt{\eta}$ for $p \in R-K$ and $=0$ for $p \in K$ is admissible for $\left\{\gamma_{K}^{1}\right\}$ and

$$
\left.\lambda i \gamma_{K}^{1}\right\}^{-1} \leqq \iint_{k} \dot{\psi}^{2} d x d y=1 / \eta \iint_{K=K} \varphi_{1}^{2} d x d y<\infty
$$

which is absurd. That is, for any given $\eta>0$ there exists a curve $\gamma_{K} \epsilon\left\{\gamma_{K}\right\}$ such that the inverse inequality of (1.15) holds. Therefore we can prove this proposition at once, q.e.d.

Corollary - If $R \in O_{G}$ and $d f$ is an Abelian differential on $R$ with finite Dirichlet integral taken over $R-K$. Then for any cycle $C \sim 0(\bmod \Im), C \subset R-K$

$$
\int_{C} d f=0
$$

Proof. Since $R \in O_{G}, \lambda\left\{C_{\}}=0\right.$ (Prop. 4), hence by Prop. 7 there exists a sequence of curves $C_{n} \epsilon\{C\}$ tending to is such that $\int_{C_{n}}|d f| \rightarrow 0 \quad(n \rightarrow \infty)$. Since $C$ is homologous to a cycle $C_{n}{ }^{\prime}$ on $C_{n}$ with bounded $(\leqq K)$ coefficients and $d f$ has no pole on $R-K$ 
On Riemann's period relations on open Riemann surfaces

$$
\left|\int_{C} d f\right|=\left|\int_{C^{\prime}{ }_{n}} d f\right| \leqq K \int_{C_{n}}|d f| \rightarrow 0 \text {, q.e.d. }
$$

5. Now we shall consider two subsets $\{\Gamma\},\left\{L_{\}_{k}}\right.$ of $\{C\}$.

(I) $\{\Gamma\}:\{\Gamma\}$ is the set of $\Gamma \in\{C\}$ such that in the decomposition of $\Gamma$ into components, i.e. $\Gamma=\sum_{i} \Gamma_{i}, \Gamma_{i} \cap \Gamma_{j}=\phi(i \neq j)$, each curve $\Gamma_{i}$ divides $R$ into two disjoint parts.

(II) $\{L\}_{E}$ : This is the system of curves of $\{\Gamma\}$ depending on an exhaustion $E=\left\{R_{n}\right\}$ such that $L_{n} \equiv \partial R_{n} \epsilon\{\Gamma\}$. That is, $\left\{L_{\xi_{E}}=\right.$ $\bigcup_{n=1}^{\infty}\left\{L_{n}\right\}$ where $\left\{L_{n}\right\}$ is the set of curves of $\{\Gamma\}$ contained in annuli including $L_{n}{ }^{5}{ }^{5}$

First of all we note $\{\Gamma\}$ and $\{L\}_{E}$ contain an infinite number of curves tending to $\mathfrak{\Im}$ (cf. Sario [18], p. 466).

Definition- We shall denote by $O^{\prime}$ or $O^{\prime \prime}$ the classes of Riemann surfaces for which $\lambda\{\Gamma\}=0$ resp. $\lambda\{L\}_{k}=0$ for certain exhaustion E.

Since $\left\{L_{\}_{E}} \subset\left\{I^{\prime}\right\} \subset\{C\}\right.$ and $\lambda\{C\}=0$ is equivalent to $R \in O_{G}$, we have $O^{\prime \prime} \subset O^{\prime} \subset O_{f_{i}}$. In the following of this paragraph we study on the properties of classes $O^{\prime}$ and $O^{\prime \prime}$.

6. The single-valued harmonic function outside of a compact set.- Let $K$ be a compact domain on $R \in O_{G ;}$ with analytic boundaries and $u$ be a single-valued harmonic function defined on $R-K{ }^{(6)}$ and bounded to one side (e.g. bounded or positive harmonic function). Then it holds for instance the following properties.

PROPOSITION 8.- (a) If $u$ is bounded, then the maximum and minimum principle hold.

$\left(\mathrm{a}^{\prime}\right)$ Maximum principle also holds even if $\lim \max u(p) / \lambda\left\{\Gamma^{n}\right\}$ $=0$. That is, if $u$ is unbounded, then $\max _{p \in \partial R_{n}}|u(p)| \geqq r_{i} \lambda\left\{\Gamma^{n} ;(\eta>0)\right.$ (Kusunoki [7]).

(b) $u$ is bounded if and only if $u$ has a finite Dirichlet integral. Moreover then $\int_{\partial K} d u^{*}=0$ (R. Nevanlinna [13], [14]).

$\left(\mathrm{b}^{\prime}\right) \quad u$ is bounded if and only if $\int_{\partial K} d u^{*}=0$.

5) By annulus including $l \in\{\Gamma\}$ we mean the union of doubly connected ring domains each of which includes a component of $l$.

6) This is not necessarily connected, but we mean hereby $R-K$ a component of it. 
Here we prove only the sufficient condition of $\left(b^{\prime}\right)$. We assume e.g. $u>-M$. Let $v_{n}$ be a harmonic function such that $v_{n}=u$ on $\partial K$ and $=-M$ on $\partial R_{n}$. Then a suitable subsequence, say $\left\{v_{n}\right\}$ tend to a bounded harmonic function $v$ which is equal ta $u$ on $\partial K$. Suppose $u$ is unbounded, then $U=u-v=\lim _{n \rightarrow \infty}\left(u-v_{n}\right) \geqq 0$ is non-constant and $=0$ on $\partial K$. Since $\int_{\partial K} d v^{*}=0$ by (b), we have $\int_{\partial K} d U^{*}=\int_{\partial K}(\partial U / \partial \nu) d s=0$. It follows $U^{*}=$ const. on $\partial K$, because $\partial U / \partial \nu=0$ on $\partial K$. Now since the curve $\partial K$ is analytic, the function $U+i U^{*}$ is also analytic on $\partial K$ by the principle of reflection, therefore $U \equiv$ const. $=0$ which is absurd, q.e.d.

Now the problem of limits (at ideal boundary) of bounded harmonic function is more complicated. Let $f$ be a real or complex valued continuous function defined on $R-K$ and $S_{\alpha}{ }^{f}$ be the set of limit values at $\alpha \in \mathfrak{F}$, i.e. $S_{\alpha}{ }^{f}=\left\{\beta ; \lim _{n \rightarrow \infty} f\left(p_{n}\right)=\beta, p_{n} \rightarrow \alpha\right\}$, then for any two equivalent sequences $\left\{\Omega_{n}\right\}$ and $\left\{\Omega_{m}{ }^{\prime}\right\}$ determining $\alpha$

$$
S_{\alpha}{ }^{f}=\bigcap_{n=1}^{\infty} \overline{f\left(\Omega_{n}\right)}=\prod_{m=1}^{\infty} \overline{f\left(\Omega_{m}{ }^{\prime}\right)} .
$$

This is a closed set. Now if $f$ is a bounded analytic function, it has always the limit, i.e. for any $\alpha \epsilon \Im, S_{\alpha}{ }^{f}$ consists of a single point. (Heins [4], A. Mori [10]). But in case of a bounded harmonic function there exists an example of Riemann surface $\epsilon O_{G}$ of infinite genus for which it does not have a limit (Heins [4]). For this problem we have the following

THEOREM 1.- Suppose $R \in O^{\prime}$ and $u(p)$ be a single-valued bounded harmonic function on $R-K$. Then $u(p)$ has always $a$ limit when $p$ tends to any ideal boundary element $\alpha$.

Proof. Since $R \in O^{\prime} \subset O_{G}$, by Prop. 8 (b) $u$ has a finite Dirichlet integral over $R-K$. Therefore by Prop. 7 there exists a sequence of curves $\Gamma_{n} \in\{\Gamma\}, n=1,2, \cdots$ tending to $\Im$ such that

$$
\int_{\Gamma_{n}}\left|w^{\prime}\right||d z|=\int_{\Gamma_{n}}|d w| \rightarrow 0 \text { for } n \rightarrow \infty,
$$

where $w=u+i u^{*}$ in $R-K,=0$ unless $u$ is defined. Now let $\left\{\Omega_{n}\right\}$ be a determining sequence of $\alpha$. Since $\Gamma_{n} \sim \partial K$ tends to $\mathfrak{J}$, there exists a number $m_{1}$ such that $\Omega_{n_{1}}\left(\equiv \Omega_{1}\right) \cup \Gamma_{m_{1}} \neq \phi$, then there exists also a number $n_{2}$ such that $\Gamma_{n_{1}} \cap \Omega_{n_{2}}=\phi$, since $\Omega_{n} \rightarrow \Im$. Now let 
On Riemann's period relations on open Riemann surfaces 11 $\Gamma_{m_{1}}^{i_{1}} \sim 0(\bmod \Im)$ be a component of $\Gamma_{m_{1}}$ which divides $C_{n_{1}}$ from $C_{n_{2}}$. We write by $\Omega_{1}{ }^{\prime}$ the non-compact domain which is bounded by the relative boundary $\Gamma_{m_{1}}^{i_{1}}$ and contains the domain $\Omega_{n_{2}}$. By the same way we determine the $\Omega_{2}^{\prime}$ such that $\Omega_{n_{2}} \supset \Omega_{2}^{\prime} \supset \Omega_{n_{3}}$. And so on. Thus we have a sequence of domains $\left\{\Omega_{m}{ }^{\prime}\right\}$ which is equivalent to $\left\{\Omega_{n}\right\}$. Because for given $i, j$ there exist $p, q$ such that $\Omega_{i} \supset \Omega_{n^{\prime}} \supset \Omega_{\eta^{\prime}}{ }^{\prime}, \Omega_{j}{ }^{\prime} \supset \Omega_{n_{j+1}} \equiv \Omega_{q}$. Therefore by (1.16) $S_{a}{ }^{u}=\bigcap_{n=1}^{\infty}$ $\overline{u\left(\Omega_{n}{ }^{\prime}\right)}$. Now by Prop. 8 (a) $u(p)$ attains to $\sup _{p \in \kappa} u(p)$ and $\inf _{p \in R-K} u(p)$ at the relative boundary $\partial K$. If $S_{\alpha}{ }^{u}$ contains two different values $a$ and $b$, then $a, b \epsilon \overline{u\left(\mathcal{S}_{n}{ }^{\prime}\right)}$ for all $n$ and

$$
|a-b| \leqq \max _{p \in L_{n}} u(p)-\min _{p \in L_{n}} u(p)
$$

where $L_{n} \equiv \Gamma_{m_{n}}^{i_{i_{n}}}$ denotes the relative boundary of $\Omega_{n}{ }^{\prime}$. Since $L_{n} \sim 0$ (mod $\Im)$ and consists of a single component, it follows

$$
\max _{p \in L_{n}} u(p)-\min _{p \in L_{n}} u(p) \leqq \int_{L_{n}}|d u| \leqq \int_{\Gamma_{m_{n}}}|d w|,
$$

hence $0<|a-b| \leqq \int_{\Gamma_{m_{n}}}|d w|$ for all $n$, which contradicts with (1.17), q.e.d.

7. Now we shall prove a sufficient condition for which $R$ should belong to class $O^{\prime \prime}$ therefore to $O^{\prime}$. Let $D_{n}, n=1,2, \cdots$ be a sequence of annuli which are disjoint each other and include the curves $L_{n}$ of $\{\Gamma\}$ and let $\left\{c_{n}\right\}$ be the set of curves of $\{\Gamma\}$ lying in $D_{n}$, then we have by Prop. 3

$$
\lambda\left\{c_{n}\right\}=2 \pi / \log \mu_{n}
$$

where $\mu_{n}$ denotes the Sario-Pfluger's ring modul of $D_{n}$. Since $D_{m} \cap D_{n}=\phi(n \neq m)$ by Prop. 2 (iii) we have

$$
\lambda\left\{\cup_{n=1}^{N}\left\{c_{n}\right\}\right\}^{-1}=\sum_{n=1}^{N} \lambda\left\{c_{n}\right\}^{-1}
$$

and by Prop. 2 (i)

Hence

$$
\lambda\{\Gamma\} \leqq \lambda\{L\}_{k} \leqq \lambda\left\{\bigcup_{n=1}^{N}\left\{c_{n}\right\}\right\} \quad \text { for any } N .
$$

$$
\frac{1}{\lambda\{\Gamma\}} \geqq \frac{1}{\lambda\{L\} E} \geqq \frac{1}{2 \pi} \log \prod_{n=1}^{N} \mu_{n} .
$$


Therefore we have

THEOREM 2.-Let $D_{n}, n=1,2, \cdots$ be a sequence of annuli which are disjoint each other and include the curves of $\{\Gamma\}$ and let $\mu_{n}$ be the Sario-Pfluger's modul of $D_{n}$. If

$$
\prod_{n=1}^{\infty} \mu_{n}=\infty
$$

then $R \in O^{\prime \prime} \subset O^{\prime}$.

COROLlaRY (Heins [4]). - Let $R$ be a parabolic Riemann surface which has only one ideal boundary element $\alpha$. Let $\left\{D_{n}\right\}$ be a sequence of doubly connected domains with analytic Jordan boundaries which are disjoint each other and $D_{n}$ separate $D_{n-1}$ from $\alpha$. If the product of modul of $D_{n}$ diverges, then every single-valued bounded harmonic function on an end (i.e. $R-K$ ) has a limit at $\alpha$.

8. Let $R \in O_{G}$ be of finite genus. Then we can take a compact domain $K$ so large that each component $B_{i}$ of $R-K$ is of planar character (schlichtartig). Therefore we can construct the (Evans) potential $U_{i}$ on $B_{i}$ such that $U_{i}(p) \rightarrow \infty$ for $p \rightarrow \mathfrak{J}, p \in B_{i}$ and $U=0$ on $\partial K, \int_{\partial K} d U^{*}=2 \pi$, where $\dot{U}=U_{i}$ in $B_{i}$. Let

$$
\lambda_{1}<\lambda_{2}<\cdots \cdots
$$

be all values of $U$ for which $U=\lambda_{i}$ contain the points where grad $U=0$, then each component of $D_{n}$ :

$$
D_{n}=\left\{p ; \lambda_{n-1}<U(p)<\lambda_{n}\right\}
$$

is a doubly connected ring domain (cf. Ahlfors [1], p. 16), moreover e.g. the level curve $L_{n}: U=\lambda_{n}{ }^{\prime}\left(\lambda_{n-1}<\lambda_{n}{ }^{\prime}<\lambda_{n}\right)$ belong to $\{\Gamma\}$, because each $B_{i}$ is of planar character. Since $\mu_{n}=\exp$. $\left(\lambda_{n}-\lambda_{n-1}\right)$, it follows $\left.\stackrel{N}{I I} \mu_{n} \rightarrow \infty \quad(N \rightarrow \infty),{ }^{7}\right)$ therefore by Theorem 2 we have $R \in O^{\prime \prime} \subset O^{\prime}$, i.e. $O_{G}=O^{\prime \prime}=O^{\prime}$. But if $R$ is of infinite genus, there exists a Riemann surface which belongs to $O_{G}$, but not $O^{\prime}$ (cf. sec. 6).

THEOREM 3.-If $R$ is of finite genus, then $O_{G}=O^{\prime}=O^{\prime \prime}$. If $R$ is of infinite genus, we have $O^{\prime \prime} \subset O_{\ddagger}^{\prime} O_{G \text {; }}$.

THEOREM 4.-Let $R, R^{\prime}$ be two Riemann surfaces, and $K, K^{\prime}$ be compact domains with analytic boundaries on $R, R^{\prime}$. Suppose that there exists $a$ one to one conformal transformation $p \leftrightarrow p^{\prime}$

7) Take $\lambda_{N+1}=\infty$ if the number of $\lambda$ 's is finite $N$. 
On Riemann's period relations on open Riemann surfaces

between the (not necessarily connected) complements $R-K$ and $R^{\prime}-$ $K^{\prime}$. Then $R \in O^{\prime}$ or $O^{\prime \prime}$ if and only if $R^{\prime} \epsilon O^{\prime}$ or $O^{\prime \prime}$ respectively.

This shows that $O^{\prime}$ or $O^{\prime \prime}$-property of Riemann surface depends only on its ideal boundary.

Proof. Under our assumption any curve $\Gamma_{K} \epsilon\{\Gamma\}, L_{K} \epsilon\{L\}_{E}$ on $R-K$ are transformed to $\Gamma_{K^{\prime}}^{\prime} \epsilon\left\{I^{\prime \prime}\right\}$, resp. $L_{K^{\prime}}^{\prime} \in\left\{L^{\prime}\right\}_{F^{\prime}}$ on $R^{\prime}-K^{\prime}$. Now if $R \in O^{\prime}$ (or $O^{\prime \prime}$ ), then $\lambda\left\{\Gamma_{K}\right\}=0\left(\lambda\left\{L_{K}\right\}_{R}=0\right)$ (Prop. 6). Since the extremal length is invariant under the conformal transformation, we have $\left.\lambda ; \Gamma_{K^{\prime}}^{\prime}\right\}=0 \quad\left(\lambda\left\{L_{K^{\prime}}^{\prime}\right\}_{E^{\prime}}=0\right)$. Therefore $R^{\prime} \in O^{\prime}$ (or $O^{\prime \prime}$ ) by Prop. 6, q.e.d.

\section{§2. Riemann's first period relation}

1. Canonical homology basis. - Let $R$ be an arbitrary Riemann surface and $\Sigma, \Sigma \prime$ denotes respectively the cell-division of $R$ and its dual subdivision. There exists a canonical homology basis $A_{1}$, $B_{1}, \cdots, A_{n}, B_{n}, \cdots$ on $R$ where $A_{n}$ belong to $\Sigma, B_{n}$ to $\Sigma^{\prime}$ and satisfy the following condition; (Ahlfors [1]).

(1) Any cycle $C$ on $R$ is expressed as

$$
C \sim \sum_{n=1}^{N}\left(p_{n} A_{n}+q_{n} B_{n}\right) \quad(\bmod \Im) .
$$

(2) The intersection numbers $N$ between them are characterized by

$$
N\left(A_{m}, A_{n}\right)=N\left(B_{m}, B_{n}\right)=0 . \quad N\left(A_{m}, B_{n}\right)=\grave{o}_{n}^{m} . \quad \text { (Kronecker). }
$$

Now let $\left\{R_{n}\right\}$ be an exhaustion of $R$. Then there exists a canonical homology basis satisfying moreover the following condition: (Ahlfors [1]).

(3) $A_{1}, B_{1}, \cdots, A_{k_{n}}, B_{k_{n}}$ are the relative homology basis of $R_{n}$ $\bmod \partial R_{n}$, i.e. any cycle $C \subset R_{n}$ is expressed as

$$
C \sim \sum_{i=1}^{k_{n_{2}}}\left(p_{i} A_{i}+q_{i} B_{i}\right) \quad\left(\bmod \partial R_{n}\right) .
$$

We shall call such basis a canonical homology basis of $\mathfrak{U}$-type with respect to $\left\{R_{n}\right\}$. For instance let $S$ be a two sheeted Riemann surface of hyperelliptic type whose branch points lie on the real positive axis $g$ and accumulate only at $\infty$. Then Fig. 1 shows a canonical basis of $\mathfrak{Q}$-type on $S$ with respect to $E=\left\{R_{u}\right\}$ such that $\partial R_{n}$ (e.g. $|z|=\gamma_{n}$ ) pass through $I_{n}$. Let $\{L\}$ be the set of analytic 


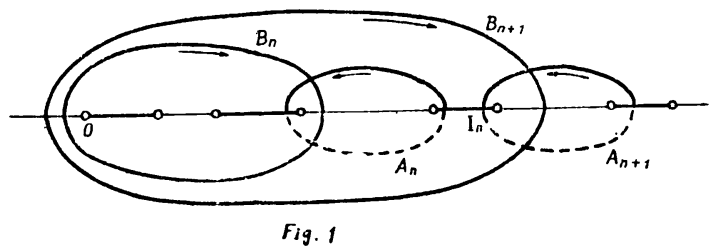

Jordan closed curves on $z$-plane which separate the circle $|z|=\boldsymbol{r}_{0}$ from $\infty$ and meet once with $g$ at slits $I_{n}$. Then it is easily seen that $\lambda\{L\}=0$ implies $\lambda\{L\}_{E}=0$. Now when $\lambda\{L\}_{F}=0$, the canonical basis of $\mathfrak{O}$-type is useful. (cf. Pfluger [17] and Th. 5,6).

2. Riemann's first period relation.- In this section we shall always consider Abelian differentials each of which has finite Dirichlet integral taken over $R$ except the neighbourhoods of a finite number of singularities. Now let $R \in O^{\prime}$, then there exists a sequence of curves $l_{\nu} \in\{\Gamma\}$ tending to $\Im$ such that they are disjoint each other and for two Abelian differentials $d f_{1}, d f_{2}$

$$
\int_{i_{\nu}}\left|d f_{1}\right| \int_{l_{\nu}}\left|d f_{2}\right| \rightarrow 0, \nu \rightarrow \infty \quad \text { (Prop. 7). }
$$

If we choose $A_{1}, B_{1}, \cdots, A_{n}, B_{n}, \cdots$ as a canonical homology basis of $\mathfrak{A}$-type with respect to this exhaustion $\left.: R^{\nu}\right\}\left(\partial R^{\nu}=l_{\nu}\right)$, then we can prove the following Riemann's relation. While, if $R \in O^{\prime \prime}$, i.e. $\lambda\{L\}_{E}=0,\{L\}_{E}=\bigcup_{n=1}^{\infty}\left\{L_{n}\right\}$ for certain exhaustion $E=\left\{R_{n}\right\}\left(\partial R_{n}=L_{n}\right)$, then under the canonical basis of $\mathfrak{A}$-type with respect to $E$ Riemann's relation holds for any two differentials. Here we shall prove for this case. The former case is proved analogously, rather more simply.

Let $A_{1}, B_{1}, \cdots, A_{n}, B_{n}, \cdots$ be the canonical basis of $\mathfrak{U}$-type with respect to $E$. Now we consider two arbitrary Abelian differentials $d f_{1}$ (1st or 2nd kind) and $d f_{2}$ which have a finite number of singularities $P_{\mu}$, where they have locally the expansions

$$
d f_{1}=\left[-\left(\frac{p a_{-p}}{z^{p+1}}+\cdots+\frac{a_{-1}}{z^{2}}\right)+a_{1}+2 a_{2} z+\cdots\right] d z
$$

$$
d f_{2}=\left[-\left(\frac{q b_{-q}}{z^{q+1}}+\cdots+\frac{b_{0}}{2}\right)+b_{1}+2 b_{2} z+\cdots\right] d z .
$$

Then we can find a sequence of curves $l_{\nu} \in\left\{L_{n_{\nu}}\right\}$ tending to $\mathfrak{\Im}$ such that they are disjoint each other and relation (2.1) holds. Let 
$A_{1}, B_{1}, \cdots, A_{k^{\nu}}, B_{k^{\nu}} \quad\left(k^{\nu} \equiv k_{n_{\nu}}\right)$ be its relative basis on $R_{n_{\nu}}$ (mod $L_{n_{\nu}}$ ), then we note that it is possible to replace $A_{i}, B_{i}$ by $A_{i}^{\prime}, B_{i}^{\prime}$ which are homologous to $A_{i}, B_{i}$ respectively, moreover $A_{i}^{\prime}, B_{i}^{\prime}$ ( $i$ $\left.=1,2, \cdots, k^{\nu}\right)$ are contained in the compact domain $R^{\nu}$ bounded by $l_{\nu}\left(\epsilon\left\{L_{n_{\nu}}\right\} \subset\{\Gamma\}\right)$. For instance in an annulus including $L_{n_{\nu}}$ and $l_{\nu}$ we replace the parts of $A_{i}, B_{i}$ lying outside of $R^{\nu}$ by its homologous counterparts in $R^{\nu}$. Now we may assume that the canonical basis are realized on the 1-dimensional elements and $\sum_{j=1}^{\lambda_{v}} a_{j}{ }^{2}=R^{v}$ where $a_{j}^{i}$ denotes $i$-dimensional element, moreover that $a_{j}{ }^{1}$ are all analytic curves not containing any pole of $d f_{1}$ and $d f_{2}$. We take $R^{v}$ so large that $R^{v} \supset R_{n o}$, where $R_{n_{0}}$ contains all singularities $P_{\mu}$ of $d f_{1}$ and $d f_{2}$. Now consider the sum of line integrals

$$
I=\sum_{j=1}^{\lambda_{2}} \int_{\partial a_{j}^{2}} f_{1} d f_{2}
$$

where the branch of $f_{1}$ is defined as follows (cf. Ahlfors [1]), i.e. for a chain (curve) $L\left(\ngtr P_{\mu}\right)$ connecting a fixed point $b_{0}\left(P_{\mu}\right)$ on $R_{u_{0}}$ to a point $b_{j}\left(\stackrel{\mp}{>} P_{\mu}\right)$ in $a_{j}^{2}$ we define by

$$
\text { (2. 4) } f_{1}\left(b_{j}\right)=\int_{L} d f_{1}+\sum_{i=1}^{k^{\nu}}\left[-N\left(A_{i}^{\prime}, L\right) \int_{B_{i}^{\prime}} d f_{1}+N\left(B_{i}^{\prime}, L\right) \int_{A_{i}^{\prime}} d f_{1}\right] \text {. }
$$

Thus defined value is independent of the choice of $L$, because the difference of two such chains forms a cycle $C$ and the difference of corresponding values of $f_{1}$ is equal to the period of $d f_{1}$ along the cycle $C+\sum_{i}\left[-N\left(A_{i}^{\prime}, C\right) B_{i}^{\prime}+N\left(B_{i}^{\prime}, C\right) A_{i}^{\prime}\right]$, which vanishes since $d f_{1}$ is of first or second kind and $C \sim \sum\left[N\left(A_{i}^{\prime}, C\right) B_{i}^{\prime}-N\left(B_{i}^{\prime}\right.\right.$, C) $\left.A_{i}^{\prime}\right]\left(\bmod l_{\nu}\right)$ (Cor. of Prop. 7). Now each $a_{j}^{1}$ inside of $R^{v}$ appears twice in these integrals (2.3) and the corresponding difference of $f_{1}$ is equal to

$$
\sum_{i}\left[-N\left(A_{i}^{\prime}, b_{j}{ }^{1}\right) \int_{B_{i}^{\prime}} d f_{1}+N\left(B_{i}^{\prime}, b_{j}{ }^{1}\right) \int_{A_{i}^{\prime}} d f_{\mathrm{r}}\right]
$$

where $b_{j}{ }_{j}^{1}$ is the dual element of $a_{j}{ }^{1}$. On the other hand

$$
\sum_{j} N\left(A_{i}^{\prime}, b_{j}^{1}\right) \int_{a_{j}^{1}} d f_{2}=\int_{A_{i}^{\prime}} d f_{2}, \quad \sum_{j} N\left(B_{i}^{\prime}, b_{j}^{1}\right) \int_{a_{j}^{1}} d f_{2}=\int_{B_{i}^{\prime}} d f_{2} .
$$

Since $A_{i}^{\prime}=A_{i}, B_{i}^{\prime}=B_{i}\left(i=1, \cdots, k_{u_{0}}\right)$ and $d f_{:}$has no pole outside of $R_{n o}$, 


$$
\int_{A_{i}^{\prime}} d f_{j}=\int_{A_{i}} d f_{j}, \int_{B_{i}^{\prime}} d f_{j}=\int_{B_{i}} d f_{j}, i=1, \cdots, k^{\downarrow}, j=1,2,
$$

hence we obtain

$$
I=\sum_{i=1}^{k \nu}\left(\int_{A_{i}} d f_{1} \int_{B_{i}} d f_{2}-\int_{A_{i}} d f_{2} \int_{B_{i}} d f_{1}\right)+\int_{l_{\nu}} f_{1} d f_{2} .
$$

Now $l_{\nu} \in\{\Gamma\}, l_{\nu}=\sum_{i} l_{\nu}{ }^{i}, l_{\nu}{ }^{i} \sim 0(\bmod \Im)$ ), therefore $\int_{l_{\nu}} d f_{2}=0$ (Cor. of

Prop. 7). Hence for fixed points $p_{i} \in l_{\nu}{ }^{i}$

therefore

$$
\left|\int_{l_{i}} f_{1} d f_{2}\right|=\left|\int_{l_{v}^{i}}\left(f_{1}(p)-f_{i}\left(p_{i}\right)\right) d f_{2}\right| \leqq \int_{l_{v}^{i}}\left|d f_{1}\right| \cdot \int_{l_{v}^{i}}\left|d f_{2}\right|,
$$

$$
\left|\sum_{i} \int_{l_{\nu}^{i}} f_{1} d f_{2}\right| \leqq \int_{l_{\nu}}\left|d f_{1}\right| \int_{l_{\nu}}\left|d f_{2}\right| \rightarrow 0 \text { for } \nu \rightarrow \infty .
$$

While, $I=2 \pi i \sum$ (residues of $f_{1} d f_{2}$ on $R^{\nu}$ ), hence we have

THEOREM 5.- For each Riemann surface $R \epsilon O^{\prime}$ there exists an exhaustion and corresponding canonical basis of $\mathfrak{M}-t y p e$ such that for two Abelian differentials $d f_{1}$ (1st or 2nd kind) and $d f_{2}$ with finite Dirichlet integrals over $R$ except the neighbourhoods of a finite number of singularities $P_{\mu}$ where they have locally the expansions $(2 \cdot 2)$, we have

(2. 5) $\lim _{\nu \rightarrow \infty} \sum_{i=1}^{k^{\nu}}\left(\int_{A_{i}} d f_{1} \int_{B_{i}} d f_{2}-\int_{A_{i}} d f_{2} \int_{B_{i}} d f_{1}\right)$

$$
=2 \pi i \sum_{P_{\mu}}\left(\sum_{n=1}^{n} n a_{-n} b_{n}-a_{0} b_{0}-\sum_{n=1}^{\eta} n a_{n} b_{-n}\right) \equiv I,
$$

where $a_{0}$ is the constant term of $f_{1}$ at $P_{\mu}$ defined by (2.4). We have $I=0$ if both $d f_{1}$ and $d f_{2}$ are of first kind. If $R \in O^{\prime \prime}$, i.e. $\lambda\{L\}_{L}=0$, then for the canonical basis of $\mathcal{A}$-type with respect to $E$ (2.5) holds always for any two such differentials." If $R$ is of finite genus, it is valid for any canonical basis on $R \in O_{G}$.

Remark. $1^{\circ}$ ) On $R \in O_{G}$ there exist Abelian differentials $d w_{i}$ (1st kind), $d t_{q}{ }^{*}$ (2nd kind, $r \geq 2$ ) with finite Dirichlet integrals

8) If necessary, we should remove $A_{i}, B_{i}$ a little so as to avoid $P_{\mu}$. 
On Riemann's period relations on open Riemann surfaces

except a neighbourhood of singularity $q$ where $d t_{q}^{r}=\left(-r / z^{r+1}+\right.$ regular term) $d z$, such that $\int_{A_{j}} d w_{i}=\hat{\boldsymbol{o}}_{i}^{j}, \int_{\hat{A}_{i}} d t_{q}^{r}=0$ (Virtanen [22]). We write here $A_{1}, B_{1}, \cdots$, as $K_{1}, K_{2}, \cdots$. If we take in Theorem 5 e.g. $d f_{1}=d w_{\mu}, d f_{2}=d w_{\nu} ; d f_{1}=d t_{q}{ }^{\prime}, d f_{2}=d w_{\nu}$, then

$$
\int_{K_{\nu}} d w_{\mu}=\int_{K_{\mu}} d w_{\nu}, \int_{K_{2 \mu}} d t_{q}{ }^{r}=\frac{-2 \pi i}{(r-1) !} \frac{d^{r} w_{\mu}(q)}{d q^{r}}, \mu, \nu=1,2, \cdots .
$$

In connexion with elementary integrals on general surface (e.g. Sario [19]) we have corresponding formulas. (cf. Schiffer-Spencer [20] p. 74-76).

$2^{\circ}$ ) Riemann's relation (2.5) holds also for certain restricted class of Abelian differentials having an infinite number of periods and singularities. For instance let $\left\{U_{n}\right\}$ be a sequence of disjoint compact domains on $R$ and $U=\bigcup_{n=1}^{\infty} U_{n}$. We consider a class of Abelian differentials which are of first or second kind and have finite Dirichlet integrals over $R-U$. Now if the extremal length vanishes for subsets of $\{\Gamma\}$ or $\{L\}_{E}$ lying on $R-U$, then by modifying Prop. 7 and its Cor. we can obtain the corresponding formula (2.5) for differentials of this class. Although it gives a relation between an infinite number of periods and singularities, there is no gurantee for the convergency of the infinite series in (2.5), but it will converge for instance under further restriction such as (3.13).

\section{§3. Riemann's second (bilinear) relation}

1. By the same way as the proof of Theorem 5 we have

TheOREM 6.-For each Riemann surface $R \in O^{\prime}$ there exists an exhaustion and corresponding canonical homology basis of $\mathfrak{A}-$ type on $R$ such that for two Abelian differentials $d f_{j}=d u_{j}+i d v_{j}(j=1,2)$ of the first kind with finite Dirichlet integrals we have

(3. 1) $\quad D_{R}\left(u_{1}, u_{2}\right)=\lim _{\nu \rightarrow \infty} \sum_{i=1}^{k \nu}\left(\int_{A_{i}} d u_{1} \int_{B_{i}} d v_{2}-\int_{B_{i}} d u_{1} \int_{A_{i}} d v_{2}\right)$.

If $R \in O^{\prime \prime}$, i.e. $\lambda\{L\}_{k}=0$, then for the canonical basis of $\mathfrak{A}$-type with respect to $E$ (3.1) holds for any two such differentials.

2. For another extension of Riemann's second relation we 
shall use the Ahlfors' theory of Schottky differentials under the same notations as Ahlfors [2].

TheOREM 7.- Let $R \in O_{H u}$ and $A_{1}, B_{1}, \cdots, A_{n}, B_{n}, \cdots$ be an arbitrary canonical homology basis on $R$ and let $d f_{j}=d u_{j}+i d v_{j}(j=$ $1,2)$ be any two Abelian differentials of the first kind with finite Dirichlet integrals. If $u_{1}$ (or $\left.v_{2}\right)$ has only a finite number of nonvanishing periods, then we have

$$
D_{k}\left(u_{1}, u_{2}\right)=\sum_{i=1}^{N}\left(\int_{A_{i}} d u_{1} \int_{B_{i}} d v_{2}-\int_{B_{i}} d u_{1} \int_{A_{i}} d v_{2}\right) .
$$

Moreover, this theorem does not hold for $R \notin O_{H D}$.

Proof. Since $\Omega_{i} \equiv d u_{i}(i=1,2)$ are harmonic differentials with finite (Dirichlet) norm, for any cycle $C \sim 0(\bmod \Im)$

$$
\int_{c} \Omega_{i}=0 \quad(i=1,2)
$$

Suppose e.g.

$$
\int_{A_{n}} \Omega_{1}=\int_{B_{n}} \Omega_{1}=0, \quad n \geqq N+1 .
$$

Now let $\left\{R_{n}\right\}$ be an arbitrary exhaustion of $R$. We take $R_{n_{0}}$ so large that it contains completely the cycles $A_{1}, B_{1}, \cdots, A_{N}, B_{N^{*}}$. We define the branch in $R_{n}\left(n>n_{0}\right)$ of $u_{1}$ as before (2.4), i.e.

$$
u_{1}\left(b_{j}\right)=\int_{L} d u_{1}+\sum_{i=1}^{N}\left[-N\left(A_{i}, L\right) \int_{B_{i}} d u_{1}+N\left(B_{i}, L\right) \int_{A_{i}} d u_{1}\right] .
$$

Under (3.3), (3.4) we find that this value is independent of the choice of $L$ connecting $b_{0}$ to $b_{j}$ as before. Now let $\tau_{n}{ }^{*} \in S_{0}{ }^{*}\left(R_{n}\right)$ be the Schottky harmonic differential on $R_{n}\left(n>n_{0}\right)$ with the same periods as $\left(\Omega_{2}{ }^{*}\right)^{*}=-\Omega_{2}$ on $R_{n}$, then $-\Omega_{2}-\tau_{n}{ }^{*}$ becomes an exact differentials, say $d V_{n}$.

$$
\begin{aligned}
\text { (3. 5) } \Omega_{2}{ }^{*} & =\tau_{n}-d V_{n}{ }^{*} . \quad \tau_{n}=0 \text { on } \partial R_{n} . \\
\text { (3. 6) } D_{R_{n}}\left(u_{1}, u_{2}\right) & =\iint_{R_{n}} \Omega_{1} \Omega_{2}{ }^{*}=\iint_{R_{n}} \Omega_{1} \tau_{n}-\iint_{R_{n}} \Omega_{1} d V_{n}{ }^{*} \equiv I_{1}^{n}+I_{2}^{n} .
\end{aligned}
$$

By Schwarz's inequality

$$
\left|I_{2}^{n}\right|^{2} \leqq\left\|\Omega_{1}\right\|_{R_{n}}^{2}\left\|d V_{n}{ }^{*}\right\|_{R_{n}}^{2} .
$$


Since $R \in O_{m}$, we have

(3. 7)

$$
\left\|d V_{n}{ }^{*}\right\|_{R_{n}}^{2}=\left\|\Omega_{2} *\right\|_{R_{n}}^{2}-\left\|\tau_{n}\right\|_{R_{n}}^{2} \rightarrow 0 \quad(n \rightarrow \infty),
$$

therefore under the condition $\left\|Q_{U_{1}}\right\|_{R}<\infty$

$$
I_{2}^{n} \rightarrow 0 \quad(n \rightarrow \infty) .
$$

On the other hand, by Green's formula and integration by parts we have

$$
I_{1}^{\prime \prime}=\sum_{j} \int_{\partial a_{j}^{2}} u_{1} \tau_{n} .
$$

Now each $a_{j}{ }^{1}$ inside of $R_{n}$ appears twice in these line integrals and the corresponding difference of $u_{1}$. is equal to

$$
\sum_{i=1}^{N}\left[-N\left(A_{i}, b_{j}^{1}\right) \int_{B_{i}} d u_{1}+N\left(B_{i}, b_{j}{ }^{1}\right) \int_{A_{i}} d u_{1}\right] .
$$

Since $R_{n} \supset R_{n 0} \supset A_{i}, B_{i}(i=1,2, \cdots, N)$, for $i=1, \cdots, N$

$$
\text { (3. 11) } \sum_{j} N\left(A_{i}, b_{j}^{1}\right) \int_{a_{j}^{1}} \tau_{n}=\int_{A_{i}} \tau_{n}, \quad \sum_{j} N\left(B_{i}, b_{j}^{1}\right) \int_{a_{j}^{1}} \tau_{n}=\int_{B_{i}} \tau_{n} .
$$

Since $\tau_{n}=0$ on $\partial R_{n}$ and $\tau_{n}$ converge uniformly to $\Omega_{2}{ }^{*}=d v_{\text {v }}$ on every compact set on $R$, we obtain the desired result (3.2) by (3.6) (3.11) when $n \rightarrow \infty$. Especially if we take $f_{1}=f_{2}=f$, then

$$
\text { (3. 12) . } \quad D_{R}(u)=\sum_{i=1}^{N}\left(\int_{A_{i}} d u \int_{B_{i}} d v-\int_{B_{i}} d u \int_{A_{i}} d v\right) \text {. }
$$

Now if this theorem holds for $R \notin O_{m}$, then for any single-valued harmonic function $u$ with finite Dirichlet integral we have $D_{R}(u)=0$ by (3.12), i.e. $u \equiv$ const., therefore $R \in O_{H}$, which is absurd, q.e.d.

Remark. - This theorem includes the Virtanen's result for $R \in O_{G}$ (Virtanen [22]). By Theorem 7 we can immediately extend the Virtanen's theory on $A$-periods to Riemann surface $\epsilon O_{m l}$ : The necessary and sufficient condition in order that there exists an Abelian integral of the first kind with finite norm and having the given $A$-periods. The existence of Abelian integral of the second kind with finite norm (except the neighbourhood of singularities) without any $A$-periods etc.

3. Let $A_{1}, B_{1}, \cdots, A_{n}, B_{n}, \cdots$ be a canonical homology basis of $\mathfrak{U}$-type with respect to an exhaustion $\left\{R_{n}\right\}$ of $R$. We denote 
$\left\{A_{i}^{(n)}\right\},\left\{B_{i}^{\prime n)}\right\}\left(1 \leqq i \leqq k_{n}\right)$ the set of cycles which are homologous to $A_{i}$ resp. $B_{i}$ and are contained in $R_{n}$. Then $\lambda\left\{A_{i}^{(n)}\right\}, \lambda\left\{B_{i}^{(n)}\right\} \geqq 0^{9)}$ are monotone decreasing for fixed $i$.

THEOREM 8.- Let $R \in O_{m D}$ and $A_{1}, B_{1}, \cdots, A_{n}, B_{n}, \cdots$ be $a$ canonical homology basis of $\mathfrak{A}$-type with respect to an exhaustion $\left\{\boldsymbol{R}_{n}\right\}$ of $R$. If for any $n$

$$
\sum_{i=1}^{k_{n}} \sqrt{\lambda\left\{A_{i}^{(n)}\right\} \lambda\left\{B_{i}^{(n)}\right\}}<M<\infty
$$

where $k_{n}$ denotes the genus of $R_{n}$, then we have for any differentials $d f_{1}, d f_{2}$ with finite normes

$$
D_{R}\left(u_{1}, u_{2}\right)=\lim _{n \rightarrow \infty} \sum_{i=1}^{k_{n}}\left(\int_{A_{i}} d u_{1} \int_{B_{i}} d v_{2}-\int_{A_{i}} d v_{2} \int_{B_{i}} d u_{1}\right) .
$$

Hence if $d u_{1}$ and $d v_{2}$ have only a finite number of non-vanishing A-periods, (3. 2) holds.

Proof. Suppose $\lambda\left\{A_{i}^{(n)}\right\}$ and $\lambda\left\{B_{i}^{(n)}\right\}>0$, then by (1.2) there exist the cycles $A_{i}{ }^{n}, B_{i}{ }^{n}$ in $R_{n}$ which are homologous to $A_{i}, B_{i}$ respectively and for given $0<\varepsilon_{n}<\min \left(\sqrt{\left.\hat{i}_{i} A_{i}^{(n)}\right\}}|| d V_{n} \|_{R_{n}}, \sqrt{\left.\lambda_{i} B_{i}^{(n)}\right\}}\right.$ $\left.\left\|d f_{1}\right\|_{R_{n}}\right)$

$$
\left.\left|\int_{B_{i}} d u_{1}\right|=\left|\int_{B_{i}^{n}} d u_{1}\right| \leqq \int_{B_{i}^{n}}\left|d f_{1}\right| \leqq \sqrt{\lambda_{i} B_{i}^{(n)}}\right\} \mid d f_{1} \|_{R}
$$

$$
\begin{aligned}
& +\varepsilon_{n}<2 \sqrt{\left.\overline{\lambda_{i}} B_{i}^{(n)}\right\}}\left\|d f_{1}\right\|_{R}, \\
& \left|\int_{A_{i}} d V_{n}{ }^{*}\right|=\left|\int_{A_{i}^{n}} d V_{n} *\right| \leqq \int_{A_{i}^{n}}\left|d w_{n}\right| \leqq \sqrt{\left.\lambda_{i} A_{i}^{(n)}\right\}} \mid d V_{u} \|_{R_{n}} . \\
& +\varepsilon_{n}<2 \sqrt{\lambda_{i} A_{i}^{(n)}} \mid\left\|d V_{n}\right\|_{R_{n}},
\end{aligned}
$$

where $d w_{n}=d V_{n}+i d V_{n}{ }^{*}$ in $R_{n}$ and $=0$ in $R-R_{n}$. These inequalities still hold even if $\lambda\left\{A_{i}^{(n)}\right\}$ (or $\left.\lambda\left\{B_{i}^{(n)}\right\}\right)=0$, because if $\left|\int_{A_{i}} d w_{n}\right|=\eta>0$, then $\varphi=\left|w_{n}{ }^{\prime}\right| / \eta$ is admissible for $\left\{A_{i}^{(n)}\right\}$ and

$$
\lambda\left\{A_{i}^{(n)}\right\}^{-1} \leqq \iint_{R} \varphi^{2} d x d y=1 / r^{2} \iint_{R_{n}}\left|w_{n}^{\prime}\right|^{2} d x d y<\infty
$$

9) The extremal length with respect to the set of cycles is analogously defined by taking the line integral along the cycle. (cf. Hersch [5]). Or, from the beginning we consider the canonical basis of $\mathfrak{A}$-type such that $A_{1}, B_{1}, \cdots$ are all Jordan closed curves. (cf. R. Nevanlinna [15]). 
On Riemann's period relations on open Riemann surfaces

i.e. $\lambda\left\{A_{i}^{(n)}\right\}>0$ which is absurd, hence $\int_{A_{i}} d V_{n}^{*}=\int_{A_{i}} d w_{n}=0$, etc. Now by (3.5) we have analogously

$$
\begin{array}{r}
D_{R_{n}}\left(u_{1}, u_{2}\right)=\sum_{i=1}^{k_{n}}\left(\int_{A_{i}} d u_{1} \int_{B_{i}} d v_{2}-\int_{B_{i}} d u_{1} \int_{A_{i}} d v_{2}\right) \\
+\sum_{i=1}^{k_{n}}\left(\int_{A_{i}} d u_{1} \int_{B_{i}} d V_{n} *-\int_{A_{i}} d V_{n} * \int_{B_{i}} d u_{1}\right) .
\end{array}
$$

Hence by (3.13), (3.14), (3.7)

$$
\left|\sum_{i=1}^{k_{n}} \int_{B_{i}} d u_{1} \int_{\dot{A}_{i}} d V_{n}^{*}\right|<4\left\|d f_{1}\right\|_{R}\left\|d V_{n}^{*}\right\|_{R_{n}} \sum_{i=1}^{k_{n}} \sqrt{\lambda\left\{A_{i}^{(n)}\right\} \lambda\left\{B_{i}^{(n)}\right\}} \rightarrow 0 \quad(n \rightarrow \infty)
$$

and analogously

$$
\left|\sum_{i=1}^{k_{n}} \int_{B_{i}} d V_{n} * \int_{A_{i}} d u_{1}\right| \rightarrow 0 \quad(n \rightarrow \infty) .
$$

Therefore we have the conclusion for $n \rightarrow \infty$, q.e.d.

On such a Riemann surface every Abelian integral of the first kind with finite norm is therefore determined except a constant by its $A$-periods. (cf. Ahlfors [1], L. Myrberg [11]).

Finally we note that the conditions in Theorems 6 and 8 are both concerned with the extremal length with respect to the set of cycles; the one is the set of cycles dividing Riemann surface, and the other the set of non dividing cycles.

$$
\begin{aligned}
& \text { Mathematical Institute } \\
& \text { Kyoto University } \\
& \text { Revised July 15, } 1956
\end{aligned}
$$

\section{REFERENCES}

[1] Ahlfors, L., Normalintegral auf offenen Riemannschen Flächen. Ann. Acad. Sci. Fenn. Ser. A. I. 35 (1947).

[2] Ahlfors, L., Open Riemann surfaces and extremal problems on compact subregion. Comm. Math. Helv. 24 (1950) pp. 100-134.

[3] Ahlfors, L., Beurling, A., Conformal invariants and function-theoretic null-sets. Acta Math. 83 (1950) pp. 101-129.

[4] Heins, M., Riemann surfaces of infinite genus. Ann. of Math. 55 (1952) pp. 296-317. 
[5] Hersch, J., Longueurs extrémales et théorie des fonctions. Comm. Math. Helv. 29 (1955) pp. 301-337.

[6] Hornich, H., Integrale erster Gattung auf speziellen transzendenten Riemannschen Flächen. Monat. für Math.-Phy. 40 (1933) pp. 241-282.

[7] Kusunoki, Y., Maximum principle for analytic functions on open Riemann surfaces. Mem. Coll. Sci. Univ. of Kyoto. 28 (1953) pp. 61-66.

[8] Kusunoki, Y., Theory of Abelian integrals (in japanese) Sûgaku, 7 (1955) pp. $32-45$.

[9] Kusunoki, Y., Some classes of Riemann surfaces characterized by the extremal length. Proc. of the Japan Acad. 32 (1956) No. 6.

[10] Mori, A., An imbedding theorem on finite covering surfaces of the Riemann sphere. Journ. Math. Soc. of Japan, 5 (1953) pp. 263-268.

[11] Myrberg, L., Normalintegrale auf zweiblättrigen Riemannschen Flächen mit reellen Verzweigungspunkten. Ann. Acad. Sci. Fenn. Ser. A. I. 71 (1950).

[12] Myrberg, P. J., Über transzendente hyperelliptische Integrale erster Gattung. Ibid. 14 (1943).

[13] Nevanlinna, R., Quadratisch integrierbare Differentiale auf einer Riemannschen Mannigfaltigkeit. Ibid. 1 (1940).

[14] Nevanlinna, R., Über das Anwachsen des Dirichletintegrals einer analytischen Funktion auf einer offenen Riemannschen Fläche. Ibid. 45 (1948).

[15] Nevanlinna, R., Uniformisierung. (1953) Berlin.

[16] Ohtsuka, M., Sur un théorème étoilé de Gross. Nagoya Math. Jour. 9 (1955) pp. 191-207.

[17] Pfluger, A., Über die Riemannsche Periodenrelation auf transzendenten hyperelliptischen Flächen. Comm. Math. Helv. 30 (1956) pp. 98-106.

[18] Sario, L., An extremal method on arbitrary Riemann surfaces. Trans. Amer. Math. Soc. 73 (1952) pp. 459-470.

[19] Sario, L., Existence des intégrales abéliennes sur les surfaces de Riemann arbitraire. C. R. Acad. Sci. Paris 229 (1949) pp. 168-170.

[20] Schiffer, M. and Spencer, D. C., Functionals of finite Riemann surfaces. Princeton (1954).

[21] Stoilow, S., Leçons sur les principles topologiques de la théorie des fonctions analytiques. Paris (1938).

[22] Virtanen, K. I., Über Abelsche Integrale auf nullberandeten Riemannschen Flächen von unendlichem Geschlecht. Ann. Acad. Sci. Fenn. Ser. A. I. 56 (1949).

[23] Virtanen, K. I., Über eine Integraldarstellung von quadratisch integrierbaren analytischen Differentialen. Ibid. 69 (1950).

[24] Weyl, H., Die Idee der Riemannschen Fläche. Leipzig (1923). 